\title{
HIGHER RANK MATRICIAL RANGES AND HYBRID QUANTUM ERROR CORRECTION
}

\author{
NINGPING CAO ${ }^{1,2}$, DAVID W. KRIBS ${ }^{1,2}$, CHI-KWONG LI ${ }^{3}$, MIKE I. NELSON ${ }^{1}$, YIU-TUNG POON ${ }^{4,6}$, \\ BEI ZENG ${ }^{1,2,5}$
}

\begin{abstract}
We introduce and initiate the study of a family of higher rank matricial ranges, taking motivation from hybrid classical and quantum error correction coding theory and its operator algebra framework. In particular, for a noisy quantum channel, a hybrid quantum error correcting code exists if and only if a distinguished special case of the joint higher rank matricial range of the error operators of the channel is non-empty. We establish bounds on Hilbert space dimension in terms of properties of a tuple of operators that guarantee a matricial range is non-empty, and hence additionally guarantee the existence of hybrid codes for a given quantum channel. We also discuss when hybrid codes can have advantages over quantum codes and present a number of examples.
\end{abstract}

\section{INTRODUCTION}

For more than a decade, numerical range tools and techniques have been applied to problems in quantum error correction, starting with the study of higher-rank numerical ranges [1, 2] and broadening and deepening to joint higher-rank numerical ranges and beyond [3, 4, 5, 6, 7, 8, 9, 10]. These efforts have made contributions to coding theory in quantum error correction and have also grown into mathematical investigations of interest in their own right. In this paper, we expand on this approach to introduce and study a higher rank matricial range motivated both by recent hybrid coding theory advances [11, 12] and the operator algebra framework for hybrid classical and quantum error correction [13, 14]. Our primary initial focus here is on a basic problem for the matricial ranges, namely, how big does a Hilbert space need to be to guarantee the existence of a non-empty matricial range of a given type, without any information on the operators and matrices outside of how many of them there are. As such, we generalize a fundamental result from quantum error correction [15, 9] to the hybrid error correction setting.

The theory of quantum error correction (QEC) originated at the interface between quantum theory and coding theory in classical information transmission and is at the heart of designing those fault-tolerant architectures [16, 17, 18, 19, 20]. It was recognized early on during these investigations that the simultaneous transmission of both quantum and classical information over a quantum channel could also be considered, most cleanly articulated in operator algebra language in [21. More recently, but still over a decade ago, the framework of "operator algebra quantum error correction" (OAQEC) [13, 14] was introduced. Motivated by a number of considerations, including a generalization of the operator quantum error correction approach [22, 23] to infinitedimensional Hilbert space [24], it was also recognized that the OAQEC approach could provide a framework for error correction of hybrid classical and quantum information, though this specific line of investigation remained dormant for lack of motivating applications at the time. Moving to the present time and over the past few years, advantages in addressing the tasks of transmitting both quantum and classical information together compared to independent solutions have been discovered, from both information theoretic and coding theoretic points of view [25, 26, 27, 28, 29, 30, 11, 12. Additionally it is felt that these hybrid codes may find applications in physical

2010 Mathematics Subject Classification. 47L90, 81P45, 81P70, 94A40, 47A12, 15A60, 81P68.

Key words and phrases. numerical range, matricial range, joint higher rank matricial ranges, quantum channel, quantum error correction, hybrid codes, operator algebra. 
descriptions of joint classical-quantum systems, in view of near-future available quantum computing devices [31] and the so-called Noisy Intermediate-Scale Quantum (NISQ) era of computing [32].

This paper is organized as follows. In the next section we introduce the joint higher rank matricial ranges and we prove the Hilbert space dimension bound result. The subsequent section considers a special case that connects the investigation with hybrid quantum error correction; specifically, for a noisy quantum channel, our formulation of the joint higher rank matricial ranges for the channel's error or "Kraus" operators leads to the conclusion that a hybrid quantum error correcting code exists for the channel if and only if one of these joint matricial ranges associated with the operators is non-empty. As a consequence of the general Hilbert space dimension bound result we establish generalizations of a fundamental early result in the theory of QEC [15, 9] to the hybrid setting. In the penultimate section we explore how hybrid error correction could provide advantages over usual quantum error correction based on this analysis. We consider a number of examples throughout the presentation and we conclude with a brief future outlook discussion.

\section{Higher Rank Matricial Ranges}

Definition 2.1. Given positive integers $m, n, p, k, K \geq 1$, let $\mathcal{P}_{K}$ be the set of $n \times K$ rank-K partial isometry matrices, so $V^{*} V=I_{K}$ for $V \in \mathcal{P}_{K}$, and let $\mathcal{D}_{p}$ be the set of diagonal matrices inside the set of $p \times p$ complex matrices $M_{p}$. Define the joint rank $(k: p)$-matricial range of an $m$-tuple of matrices $\mathbf{A}=\left(A_{1}, \ldots, A_{m}\right) \in M_{n}^{m}$ by

$$
\Lambda_{(k: p)}(\mathbf{A})=\left\{\left(D_{1}, \ldots, D_{m}\right) \in \mathcal{D}_{p}^{m}: \exists V \in \mathcal{P}_{k p} \text { such that } V^{*} A_{j} V=D_{j} \otimes I_{k} \text { for } j=1, \ldots, m\right\} .
$$

Observe that when $p=1, \Lambda_{(k: p)}(\mathbf{A})$ becomes the rank- $k$ (joint when $m \geq 2$ ) numerical range considered in [1, 2, 3, 4, 5, 6, 7, 9, 10, and elsewhere.

We first discuss two reductions that we can make without loss of generality.

Remark 2.2. Since every $A \in M_{n}$ has a Hermitian decomposition $A=A_{1}+i A_{2}$, with $A_{1}, A_{2} \in H_{n}$, the set of $n \times n$ Hermitian matrices, we only need to consider $\Lambda_{(k: p)}(\mathbf{A})$ for $\mathbf{A} \in H_{n}^{m}$, where $H_{n}^{m}$ is the set of $m$-tuples of $n \times n$ Hermitian matrices.

Furthermore, suppose $T=\left(t_{i j}\right) \in M_{m}$ is a real invertible matrix, and $\left(c_{1}, \ldots, c_{m}\right) \in \mathbf{R}^{1 \times m}$. Let $\tilde{\mathbf{A}}=\left(\tilde{A}_{1}, \ldots, \tilde{A}_{m}\right)$, where for $j=1, \ldots, m$,

$$
\tilde{A}_{j}=\sum_{\ell=1}^{m} t_{\ell, j} A_{\ell}+c_{j} I_{n} .
$$

Then one readily shows that $\left(D_{1}, \ldots, D_{m}\right) \in \Lambda_{(k: p)}(\mathbf{A})$ if and only if $\left(\tilde{D}_{1}, \ldots, \tilde{D}_{m}\right) \in \Lambda_{(k: p)}(\tilde{\mathbf{A}})$, where $\tilde{D}_{j}=\sum_{\ell=1}^{m} t_{\ell, j} D_{\ell}+c_{j} I_{k}$ for $j=1, \ldots, m$. So, the geometry of $\Lambda_{(k: p)}(\mathbf{A})$ is completely determined by $\Lambda_{(k: p)}(\tilde{\mathbf{A}})$.

Now, we can choose a suitable $T=\left(t_{i j}\right)$ and $\left(c_{1}, \ldots, c_{m}\right)$ so that $\left\{\tilde{A}_{1}, \ldots, \tilde{A}_{r}, I_{n}\right\}$ is linearly independent, and $\tilde{A}_{r+1}=\cdots=\tilde{A}_{m}=0_{n}$. Then the geometry of $\Lambda_{(k: p)}(\tilde{\mathbf{A}})$ is completely determined by $\Lambda_{(k: p)}\left(\tilde{A}_{1}, \ldots, \tilde{A}_{r}\right)$. Hence, in what follows, we always assume that $\left\{A_{1}, \ldots, A_{m}, I_{n}\right\}$ is linearly independent.

The result we prove below is a generalization of the main result from [15], which applies to the $p=1$ case in our notation. This was also proved in [9] via a matrix theoretic approach and we make use of this in our proof, so we state the original result as it was presented in [9] using the present notation.

Lemma 2.3. Let $\mathbf{A}=\left(A_{1}, \ldots, A_{m}\right) \in H_{n}^{m}$ and let $m \geq 1$ and $k>1$. If

$$
n \geq(k-1)(m+1)^{2},
$$

then $\Lambda_{(k: 1)}(\mathbf{A}) \neq \emptyset$. 
Observe that if $\left(a_{1}, \ldots, a_{m}\right) \in \Lambda_{k p}(\mathbf{A})$, then $\left(a_{1} I_{p}, \ldots, a_{m} I_{p}\right) \in \Lambda_{(k: p)}(\mathbf{A})$. Thus by Lemma 2.3. if $n \geq(k p-1)(m+1)^{2}$, then $\Lambda_{k p}(\mathbf{A}) \neq \emptyset$; hence $\Lambda_{(k: p)}(\mathbf{A}) \neq \emptyset$. The following theorem gives an improvement on this bound.

Theorem 2.4. Let $\mathbf{A}=\left(A_{1}, \ldots, A_{m}\right) \in H_{n}^{m}$ and let $m, p \geq 1$ and $k>1$. If

$$
n \geq(m+1)((m+1)(k-1)+k(p-1)),
$$

then $\Lambda_{(k: p)}(\mathbf{A}) \neq \emptyset$.

Proof. We will prove the result by induction on $p$. When $p=1$, the bound $(m+1)((m+1)(k-$ $1)+k(p-1))=(k-1)(m+1)^{2}$ is given in Lemma 2.3.

Suppose $p>1$. We suppose for $r<p$, we can find an $n \times r k$ matrix $U_{r}$ and $r \times r$ diagonal matrices $D_{j, r}, 1 \leq j \leq m$ such that $U_{r}^{*} U_{r}=I_{r k}$ and $U_{r}^{*} A_{j} U_{r}=D_{j, r} \otimes I_{k}$ for all $1 \leq j \leq m$.

Since $(m+1)((m+1)(k-1)+k(p-1))>(k-1)(m+1)^{2}$, there exist an $n \times k$ matrix $U_{1}$ and scalars $d_{j}, 1 \leq j \leq m$ such that $U_{1}^{*} U_{1}=I_{k}$ and $U_{1}^{*} A_{j} U_{1}=d_{j} I_{k}$ for all $1 \leq j \leq m$. Let $U$ be unitary with first $\ell$ columns containing the columns spaces of $U_{1}, A U_{1}, \ldots, A_{m} U_{1}$. Then $\ell \leq(m+1) k$ and $U^{*} A_{j} U=B_{j} \oplus C_{j}$ with $B_{j} \in M_{\ell}$ for $j=1, \ldots, m$, and $C_{j} \in M_{n-\ell}$, where $n-\ell \geq(m+1)((m+1)(k-1)+k(p-2))$. By induction assumption, $\Lambda_{(k: p-1)}\left(C_{1}, \ldots, C_{m}\right)$ is non-empty, say, containing an $m$-tuple of diagonal matrices $\left(D_{j 1}, \ldots, D_{j m}\right) \in M_{p-1}^{m}$. So, we can find an $n \times(k-1)(m+1)^{2}$ matrix $U_{2}$ such that $U_{2}^{*} A_{j} U_{2}=D_{j \ell} \otimes I_{k}$ for $j=1, \ldots, m$. Thus, there is $V=\left[U_{1} \mid V_{2}\right] \in M_{n, p k}$ such that $V^{*} V=I_{p k}$ and $V^{*} A_{j} V=d_{j} I_{k} \oplus D_{j \ell} \otimes I_{k}$ for $j=1, \ldots, m$. Hence, $\Lambda_{(k: p)}(\mathbf{A}) \neq \emptyset$.

Remarks 2.5. (i) Let $n(k, m)$ (respectively, $n(k: p, m)$ ) be the minimum number such that for all $n \geq n(k, m)$ (respectively, $n(k: p, m)$ ), we have $\Lambda_{k}(\mathbf{A}) \neq \emptyset$ (respectively, we have $\Lambda_{(k: p)}(\mathbf{A}) \neq \emptyset$ ) for all $\mathbf{A} \in H_{n}^{m}$. Clearly, we have $n(k p, m) \geq n(k: p, m) \geq k p$. In Example 3.4 and 3.6, we will see that sometimes the lower bound can be attained.

(ii) The upper bound $(m+1)((m+1)(k-1)+k(p-1)) \geq n(k: p, m)$ in Theorem 2.4 is not optimal. The same proof shows that $n(k: p, m) \leq n(k, m)+(m+1) k(p-1)$. So, if we can lower the bound for $n(k, m)$, then we can lower the bound for $n(k: p, m)$. For example, since $n(k, 1)=2 k-1$ [2] and $n(k, 2)=3 k-2$ [9], we have $n(k: p, 1) \leq 2 p k-1$ and $n(k: p, 2) \leq 3 p k-2$. We also note that using Fan and Pall's interlacing theorem [33], one can show that $n(k: p, 1)=(p+1) k-1$.

(iii) In the proof of Theorem 2.4, suppose $U_{1}^{*} A_{j} U_{1}$ has leading $k \times k$ submatrix equal to $a_{j 1} I_{k}$. Then we can find a unitary $X$ such that $X^{*} A_{j} X=\left(B_{p q}^{(j)}\right)_{1 \leq p, q \leq 2}$ with $B_{11}^{(j)}=a_{j} I_{k}, B_{12}^{(j)}=0_{k \times r}$ and $B_{13}^{(j)}$ is $k \times s$ with $s \leq m k$. That is why we can induct on the leading $(n-s) \times(n-s)$ matrices. Of course, we can have some savings if $s<m k$ at any step.

(iv) Also, when $m=1$, it does not matter whether we want $D_{j} \otimes I_{k}$ or $C_{j} \otimes I_{k}$ for diagonal $D_{j}$ or general Hermitian $C_{j}$. We can diagonalize $C_{j}$. Note that if $n=(p+1) k-1$, then the set $\Lambda_{(k: p)}(A)$ is unique if the eigenvalues of $A$ are distinct. It should be possible to say more if there are repeated eigenvalues, and in that case one can lower the requirement of $n \geq(p+1) k-1$.

$(v)$ When $\left\{A_{1}, \ldots, A_{m}\right\}$ is a commuting family, then $A_{p}+i A_{q}$ is normal for any $p<q$. The results in 34 might be useful to study this further.

(vi) One could also study a more general class of matricial ranges in which Definition 2.1] would be viewed as a special case; namely, the definition could be broadened to allow for arbitrary $p \times p$ matrices in the $m$-tuples of $\Lambda_{(k: p)}(\mathbf{A})$, removing the diagonal matrix restriction. One can generalize Theorem 2.4 and obtain other interesting results; see Section 5.

Using similar techniques, we may also apply recent related work on the shape of joint matricial ranges [10] to obtain the following.

Theorem 2.6. Let $\mathbf{A}=\left(A_{1}, \ldots, A_{m}\right) \in H_{n}^{m}$, and $m, p, k \geq 1$ satisfy $n \geq(k p(m+2)-1)(m+1)^{2}$. Then $\Lambda_{(k p(m+2): 1)}(\mathbf{A})$ is non-empty, and $\Lambda_{(k: p)}(\mathbf{A})$ is star-shaped with star center $\left(a_{1} I_{p}, \ldots, a_{m} I_{p}\right)$ 
for any $\left(a_{1}, \ldots, a_{m}\right) \in \Lambda_{(k p(m+2): 1)}(\mathbf{A})$, i.e., $t\left(a_{1} I_{p}, \ldots, a_{m} I_{p}\right)+(1-t)\left(B_{1}, \ldots, B_{m}\right) \in \Lambda_{(k: p)}(\mathbf{A})$ for any $t \in[0,1]$ and $\left(B_{1}, \ldots, B_{m}\right) \in \Lambda_{(k: p)}(\mathbf{A})$.

\section{Application to Hybrid Quantum Error Correction}

In quantum information, a quantum channel corresponds to a completely positive and trace preserving (CPTP) linear map $\Phi: M_{n} \rightarrow M_{n}$. By the structure theory of such maps [35], there is a finite set $E_{1}, \cdots \in M_{n}$ with $\sum_{j} E_{j}^{*} E_{j}=I_{n}$ such that for all $\rho \in M_{n}$,

$$
\Phi(\rho)=\sum_{j} E_{j} \rho E_{j}^{*} .
$$

These operators are typically referred to as the Kraus operators for $\Phi$ [36], and the minimal number of operators $E_{j}$ required for this operator-sum form of $\Phi$ is called the Choi rank of $\Phi$, as it is equal to the rank of the Choi matrix for $\Phi$ [35]. In the context of quantum error correction, $E_{j}$ are viewed as the error operators for the physical noise model described by $\Phi$.

The OAQEC framework [13, 14] relies on the well-known structure theory for finite-dimensional von Neumann algebras (equivalently $\mathrm{C}^{*}$-algebras) when applied to the finite-dimensional setting [37. Specifically, codes are characterized by such algebras, which up to unitary equivalence can be uniquely written as $\mathcal{A}=\oplus_{i}\left(I_{m_{i}} \otimes M_{n_{i}}\right)$. Any $M_{n_{i}}$ with $n_{i}>1$ can encode quantum information; which when $m_{i}=1$ corresponds to a standard (subspace) error-correcting code [16, 17, 18, 19, 20] and when $m_{i}>1$ corresponds to an operator quantum error-correcting subsystem code [22, 23]. If there is more than one summand in the matrix algebra decomposition for $\mathcal{A}$, then the algebra is a hybrid of classical and quantum codes. It has been known for some time that algebras can be used to encode hybrid information in this way [21, and OAQEC provides a framework to study hybrid error correction in depth. Of particular interest here, we draw attention to the recent advance in coding theory for hybrid error-correcting codes [11], in which explicit constructions have been derived for a distinguished special case of OAQEC discussed in more detail below.

In the Schrödinger picture for quantum dynamics, an OAQEC code is explicitly described as follows: $\mathcal{A}$ is correctable for $\Phi$ if there is a CPTP map $\mathcal{R}$ such that for all density operators $\rho_{i} \in M_{n_{i}}, \sigma_{i} \in M_{m_{i}}$ and probability distributions $p_{i}$, there are density operators $\sigma_{i}^{\prime}$ such that

$$
(\mathcal{R} \circ \Phi)\left(\sum_{i} p_{i}\left(\sigma_{i} \otimes \rho_{i}\right)\right)=\sum_{i} p_{i}\left(\sigma_{i}^{\prime} \otimes \rho_{i}\right) .
$$

This condition is perhaps more cleanly phrased in the corresponding Heisenberg picture as follows: $\mathcal{A}$ is correctable for $\Phi$ if there is a channel $\mathcal{R}$ such that for all $X \in \mathcal{A}$,

$$
\left(\mathcal{P}_{\mathcal{A}} \circ \Phi^{\dagger} \circ \mathcal{R}^{\dagger}\right)(X)=X
$$

where $\Phi^{\dagger}$ is the Hilbert-Schmidt dual map (i.e., $\left.\operatorname{tr}(X \Phi(\rho))=\operatorname{tr}\left(\Phi^{\dagger}(X) \rho\right)\right)$ and $\mathcal{P}_{\mathcal{A}}(\cdot)=P_{\mathcal{A}}(\cdot) P_{\mathcal{A}}$ with $P_{\mathcal{A}}$ the unit projection of $\mathcal{A}$.

From [13, 14, we have the following useful operational characterization of correctable algebras in terms of the Kraus operators for the channel:

Lemma 3.1. An algebra $\mathcal{A}$ is correctable for a channel $\Phi(\rho)=\sum_{i} E_{i} \rho E_{i}^{*}$ if and only if

$$
\left[P E_{i}^{*} E_{j} P, X\right]=0 \quad \forall X \in \mathcal{A},
$$

where $P$ is the unit projection of $\mathcal{A}$.

In other words, $\mathcal{A}$ is correctable for $\Phi$ if and only if the operators $P E_{i}^{*} E_{j} P$ belong to the commutant $P \mathcal{A}^{\prime} P=P \mathcal{A}^{\prime}=\mathcal{A}^{\prime} P$. Applied to the familiar case of standard quantum error correction, with $\mathcal{A}=M_{k}$ for some $k$, we recover the famous Knill-Laflamme conditions [20]: $\left\{P E_{i}^{*} E_{j} P\right\}_{i, j} \subseteq \mathbb{C} P$. The result applied to the case $\mathcal{A}=I_{m} \otimes M_{k}$ yields the testable conditions from operator quantum 
error correction [22, 23]: $\left\{P E_{i}^{*} E_{j} P\right\}_{i, j} \subseteq M_{m} \otimes I_{k}$. Anything else involves direct sums and has a hybrid classical-quantum interpretation as noted above.

We next turn to the distinguished special hybrid case noted above. First some additional notation: we shall assume all our channels act on a Hilbert space $\mathcal{H}$ of dimension $n \geq 1$, and so we may identify $\mathcal{H}=\mathbb{C}^{n}$ and let $\left\{\left|e_{i}\right\rangle\right\}$ be the canonical orthonormal basis. Our algebras $\mathcal{A}$ then are subalgebras of the set of all linear operators $\mathcal{L}(\mathcal{H})$ on $\mathcal{H}$, which in turn is identified with $M_{n}$ through matrix representations in the basis $\left|e_{i}\right\rangle$. We shall go back and forth between these operator and matrix perspectives as convenient.

As in [11], consider the case that $\mathcal{A}=\oplus_{r} \mathcal{A}_{r}$ with each $\mathcal{A}_{r}=M_{k}$ for some fixed $k \geq 1$. Let us apply the conditions of Eq. (2) to such algebras. Let $P_{r}$ be the (rank $k$ ) projection of $\mathcal{H}$ onto the support of $\mathcal{A}_{r}$, so that the $P_{r}$ project onto mutually orthogonal subspaces and $P=\sum_{r} P_{r}$ is the unit projection of $\mathcal{A}=\oplus_{r} P_{r} \mathcal{L}(\mathcal{H}) P_{r}$. Observe here the commutant of $\mathcal{A}$ satisfies: $P \mathcal{A}^{\prime} P=\oplus_{r} \mathbb{C} P_{r}$. Thus by Lemma 3.1, it follows that $\mathcal{A}$ is correctable for $\Phi$ if and only if for all $i, j$ there are scalars $\lambda_{i j}^{(r)}$ such that

$$
P E_{i}^{*} E_{j} P=\sum_{r} \lambda_{i j}^{(r)} P_{r}
$$

which is equivalent to the equations:

$$
P_{r} E_{i}^{*} E_{j} P_{s}=\delta_{r s} \lambda_{i j}^{(r)} P_{r} \quad \forall r, s .
$$

Indeed, these are precisely the conditions derived in [11] (see Theorem 4 of [11]).

For what follows, let $\mathcal{V}_{r}, 1 \leq r \leq p$ be mutually orthogonal $k$-dimensional subspaces of $\mathbb{C}^{n}$ and $P_{r}$ the orthogonal projection of $\mathbb{C}^{n}$ onto $\mathcal{V}_{r}$, for $1 \leq r \leq p$. Following [11], we say that $\left\{\mathcal{V}_{r}: 1 \leq i \leq p\right\}$ is a hybrid $(k: p)$ quantum error correcting code for the quantum channel $\Phi$ if for all $i, j$ and all $r$ there exist scalars $\lambda_{i j}^{(r)}$ such that Eqs. (3) are satisfied.

Consideration of the matricial ranges defined above is motivated by the following fact, which can be readily verified from Eqs. (44).

Lemma 3.2. A quantum channel $\Phi$ as defined in Eq. (11) has a hybrid error correcting code of dimensions $(k: p)$ if and only if

$$
\Lambda_{(k: p)}\left(E_{1}^{*} E_{1}, E_{1}^{*} E_{2}, \ldots, E_{r}^{*} E_{r}\right) \neq \emptyset .
$$

We note that given a rank- $k p$ projection, with say $P=\sum_{i=1}^{k p}\left|e_{i}\right\rangle\left\langle e_{i}\right|$, and diagonal matrices $D_{j}$ that make $\Lambda_{(k: p)}$ nonempty, we may define the desired projections for $1 \leq r \leq p$, by $P_{r}=$ $\sum_{i=1}^{k}\left|e_{(r-1) k+i}\right\rangle\left\langle e_{(r-1) k+i}\right|$.

Theorem 3.3. Let $\Phi$ be a quantum channel as defined in Eq. (1) with Choi rank equal to $c$. Then $\Phi$ has a hybrid error correcting code of dimensions $(k: p)$ if

$$
\operatorname{dim} \mathcal{H} \geq c^{2}\left(c^{2}(k-1)+k(p-1)\right) .
$$

Proof. Suppose $\left\{E_{1}, \ldots, E_{c}\right\}$ is a minimal set of Kraus operators that implement $\Phi$ as in (1). For $1 \leq j<\ell \leq c$, let $F_{j \ell}=\frac{1}{2}\left(E_{j}^{*} E_{\ell}+E_{\ell}^{*} E_{j}\right)$ and $F_{\ell j}=\frac{1}{2 i}\left(E_{j}^{*} E_{\ell}-E_{\ell}^{*} E_{j}\right)$. Also, let $F_{j j}=E_{j}^{*} E_{j}$ for $1 \leq j \leq c$. Since $\sum_{j=1}^{c} E_{j}^{*} E_{j}=I$, the operator subspace $\operatorname{span}\left\{F_{j \ell}: 1 \leq j, \ell \leq c\right\}$ has a basis $\left\{A_{0}=I, \ldots, A_{m}\right\}$ with $m \leq c^{2}-1$. The result now follows from an application of Theorem 2.4.

Theorem 3.3 is useful if we have no information about the $E_{i}^{\prime} \mathrm{s}$, except the number $c$. If the $E_{i}^{\prime} \mathrm{s}$ are given, we may get a hybrid code even when $n$ is lower than the bound given in Theorem 2.4 or 3.3. The saving can come from two sources: 1) The subspace spanned by $\left\{E_{i}^{*} E_{j}: 1 \leq i, j \leq c\right\}$ can have dimension (over $\mathbb{R}$ ) smaller than $c^{2}$ in particular when restricted to the code, or 2) the operators $\left\{E_{i}^{*} E_{j}\right\}$ have some specific structures. We give some examples to demonstrate this. 
Example 3.4. Consider the error model on a three-qubit system

$$
\Phi(\rho)=p\left(X_{2} \rho X_{2}\right)+(1-p) \rho,
$$

where $X_{2}=I \otimes X \otimes I$ and $X$ is the Pauli bit flip operator and $0<p<1$ is some fixed probability. It is not hard to see that the codes $C_{1}=\operatorname{span}\{|000\rangle,|001\rangle\}$ and $C_{2}=\operatorname{span}\{|100\rangle,|101\rangle\}$ together form a correctable hybrid code for $\Phi$. One would seek to examine the matricial range

$$
\Lambda_{(k: p)}\left(E_{1}^{*} E_{1}, E_{1}^{*} E_{2}, E_{2}^{*} E_{1}, E_{2}^{*} E_{2}\right)=\Lambda_{(k: p)}\left(I, X_{2}, X_{2}, I\right) .
$$

By the above reduction to linearly independent sets of Kraus operators, we would be interested in the geometry of $\Lambda_{(k: p)}\left(X_{2}\right)$. Since $X_{2}$ is unitarily similar to $I_{4} \oplus-I_{4}, \Lambda_{(4: 2)}\left(X_{2}\right)=\{\operatorname{diag}(1,-1)\}$. Thus, for this example, we have $m=1, k=4, p=2, n=8$ and $c=2$.

Example 3.5. Consider the quantum channel on a three-qubit system given by

$$
\Phi(\rho)=p_{0} \rho+p_{1} X^{\otimes 3} \rho X^{\otimes 3^{*}}+p_{2} Y^{\otimes 3} \rho Y^{\otimes 3^{*}}+p_{3} Z^{\otimes 3} \rho Z^{\otimes 3^{*}},
$$

where $p_{0}, \ldots, p_{3}$ are probabilities summing to 1 and $X^{\otimes 3}=X \otimes X \otimes X$ etc, with the Pauli matrices $X, Y, Z$.

In this case the relevant operators $E_{i}^{*} E_{j}$ form the 3 -tuple $\left(X^{\otimes 3}, Y^{\otimes 3}, Z^{\otimes 3}\right)$, and we set $m=3$, $k=4, p=1$. Defining a partial isometry $V: \mathbb{C}^{4} \rightarrow \mathbb{C}^{2} \otimes \mathbb{C}^{2} \otimes \mathbb{C}^{2}$ by

$$
V=|000\rangle\langle 00|+| 011\rangle\langle 01|+| 101\rangle\langle 10|+| 110\rangle\langle 11|,
$$

one can verify that $V^{*} V=I_{4}$ and

$$
\begin{aligned}
V^{*}\left(X^{\otimes 3}, Y^{\otimes 3}, Z^{\otimes 3}\right) V & =\left(0_{2} \otimes I_{2}, 0_{2} \otimes I_{2}, I_{2} \otimes I_{2}\right) \\
& =\left(0_{4}, 0_{4}, I_{4}\right) .
\end{aligned}
$$

Therefore, $\Lambda_{4}\left(X^{\otimes 3}, Y^{\otimes 3}, Z^{\otimes 3}\right) \neq \emptyset$. However, $\Lambda_{(4: 2)}\left(X^{\otimes 3}, Y^{\otimes 3}, Z^{\otimes 3}\right)=\emptyset$ because $X^{\otimes 3}$ and $Y^{\otimes 3}$ do not commute.

Example 3.6. Extend the previous example to a four-qubit system given by

$$
\Psi(\rho)=p_{0} \rho+p_{1} X^{\otimes 4} \rho X^{\otimes 4^{*}}+p_{2} Y^{\otimes 4} \rho Y^{\otimes 4^{*}}+p_{3} Z^{\otimes 4} \rho Z^{\otimes 4^{*}},
$$

where $p_{0}, \ldots, p_{3}$ are probabilities summing to 1 .

In this case the relevant operators $E_{i}^{*} E_{j}$ form the 3 -tuple $\left(X^{\otimes 4}, Y^{\otimes 4}, Z^{\otimes 4}\right)$, and we set $m=3$. We are going to show that there is a unitary matrix $U \in M_{16}$ such that

$$
U^{*} X^{\otimes 4} U=D_{X} \otimes I_{4}, \quad U^{*} Y^{\otimes 4} U=D_{Y} \otimes I_{4}, \quad U^{*} Z^{\otimes 4} U=D_{Z} \otimes I_{4},
$$

for some diagonal matrices $D_{X}, D_{Y}, D_{Z} \in M_{4}$. Hence, we will have $\Lambda_{(4: 4)}\left(X^{\otimes 4}, Y^{\otimes 4}, Z^{\otimes 4}\right) \neq \emptyset$. In this case, $k=4, p=4$ and $n=16=k p$. Thus, the smallest possible $n$ is also achieved.

For $J=\left(j_{1} j_{2} j_{3} j_{4}\right) \in\{0,1\}^{4}$, let $|J\rangle=\left|j_{1} j_{2} j_{3} j_{4}\right\rangle$ and $|J|=\sum_{i=1}^{4} j_{i}$. Since $Y_{4}|J\rangle=(-1)^{|J|} X_{4}|J\rangle$, we have

$$
\begin{aligned}
X_{4}\left(|J\rangle+X_{4}|J\rangle\right) & =|J\rangle+X_{4}|J\rangle \\
X_{4}\left(|J\rangle-X_{4}|J\rangle\right) & =-\left(|J\rangle-X_{4}|J\rangle\right) \\
Y_{4}\left(|J\rangle+X_{4}|J\rangle\right) & = \begin{cases}|J\rangle+X_{4}|J\rangle & \text { if }|J| \text { is even } \\
-\left(|J\rangle+X_{4}|J\rangle\right) & \text { if }|J| \text { is odd }\end{cases} \\
Y_{4}\left(|J\rangle-X_{4}|J\rangle\right) & = \begin{cases}-\left(|J\rangle-X_{4}|J\rangle\right) & \text { if }|J| \text { is even } \\
\left(|J\rangle-X_{4}|J\rangle\right) & \text { if }|J| \text { is odd }\end{cases}
\end{aligned}
$$


Define a unitary matrix $U=\frac{1}{2}\left[u_{1} \cdots u_{16}\right]$ with columns given by

$$
\begin{aligned}
& u_{1}=(|0000\rangle+|1111\rangle)+(|0011\rangle+|1100\rangle) \\
& u_{2}=(|0000\rangle+|1111\rangle)-(|0011\rangle+|1100\rangle) \\
& u_{3}=(|0101\rangle+|1010\rangle)+(|0110\rangle+|1001\rangle) \\
& u_{4}=(|0101\rangle+|1010\rangle)-(|0110\rangle+|1001\rangle) \\
& u_{5}=(|0001\rangle+|1110\rangle)+(|0010\rangle+|1101\rangle) \\
& u_{6}=(|0001\rangle+|1110\rangle)-(|0010\rangle+|1101\rangle) \\
& u_{7}=(|0100\rangle+|1011\rangle)+(|0111\rangle+|1000\rangle) \\
& u_{8}=(|0100\rangle+|1011\rangle)-(|0111\rangle+|1000\rangle) \\
& u_{9}=(|0000\rangle-|1111\rangle)+(|0011\rangle-|1100\rangle) \\
& u_{10}=(|0000\rangle-|1111\rangle)-(|0011\rangle-|1100\rangle) \\
& u_{11}=(|0101\rangle-|1010\rangle)+(|0110\rangle-|1001\rangle) \\
& u_{12}=(|0101\rangle-|1010\rangle)-(|0110\rangle-|1001\rangle) \\
& u_{13}=(|0001\rangle-|1110\rangle)+(|0010\rangle-|1101\rangle) \\
& u_{14}=(|0001\rangle-|1110\rangle)-(|0010\rangle-|1101\rangle) \\
& u_{15}=(|0100\rangle-|1011\rangle)+(|0111\rangle-|1000\rangle) \\
& u_{16}=(|0100\rangle-|1011\rangle)-(|0111\rangle-|1000\rangle)
\end{aligned}
$$

Since, $Z_{4}=X_{4} Y_{4}$, by (的), we have (15) with

$$
D_{X}=\operatorname{diag}(1,1,-1,-1), D_{Y}=\operatorname{diag}(1,-1,-1,1) \text { and } D_{Z}=\operatorname{diag}(1,-1,1,-1) .
$$

Remark 3.7. More generally, one can consider the class of correlation channels studied in [38], which has error operators $X^{\otimes n}, Y^{\otimes n}, Z^{\otimes n}$ normalized with probability coefficients. It is proved there that when $n$ is odd, $\Lambda_{2^{n-1}}\left(X^{\otimes n}, Y^{\otimes n}, Z^{\otimes n}\right) \neq \emptyset$. Thus $n$ qubit codewords encode $(n-1)$ data qubits when $n$ is odd. When $n$ is even, it follows that $\Lambda_{2^{n-2}}\left(X^{\otimes n}, Y^{\otimes n}, Z^{\otimes n}\right) \neq \emptyset$. Using a proof similar to the above example, we can show that $\Lambda_{\left(2^{n-2}: 4\right)}\left(X^{\otimes n}, Y^{\otimes n}, Z^{\otimes n}\right)=\left\{\left(D_{X}, D_{Y}, D_{Z}\right)\right\}$, with $D_{X}, D_{Y}, D_{Z}$ given by (77). It has been proven in [38] that for $n$ even, $\Lambda_{2^{n-1}}\left(X^{\otimes n}, Y^{\otimes n}, Z^{\otimes n}\right)=\emptyset$. Actually, we can show that $\Lambda_{k}\left(X^{\otimes n}, Y^{\otimes n}, Z^{\otimes n}\right)=\emptyset$ for all $k>2^{n-2}$. Therefore, we can encode at most $n-2$ qubits. Using the hybrid code, we can get 2 additional classical bits. Very recently, this scheme has been implemented using IBM's quantum computing framework qiskit [39].

\section{Exploring Advantages of Hybrid Quantum Error Correction}

A straightforward way to form hybrid codes is to use quantum codes to directly transmit classical information. However, it is impractical since quantum resources are more expensive than classical resources. Thus, realistically, hybrid codes are more interesting when the simultaneous transmission 
of classical information and quantum information do possess advantages. One of such situations is, with a fixed set of operators $\mathbf{A}$, hybrid quantum error correcting codes exist but the corresponding quantum codes do not exist for the same system dimension $n$, i.e. $\Lambda_{(k: p)}(A) \neq \emptyset$ and $\Lambda_{k p}(A)=\emptyset$.

Proposition 4.1. Suppose $A$ is an $n \times n$ Hermitian matrix with eigenvalues $a_{1} \geq a_{2} \geq \cdots \geq a_{n}$. Then

$$
\begin{aligned}
\Lambda_{k p}(A) & =\left\{t: a_{n+1-k p} \leq t \leq a_{k p}\right\} \\
\Lambda_{(k: p)}(A) & =\left\{\left(t_{1}, \ldots, t_{p}\right): a_{i k} \leq t_{[i]} \leq a_{n+1-(p-i+1) k} \text { for } 1 \leq i \leq p\right\},
\end{aligned}
$$

where here, $t_{[1]} \geq t_{[2]} \geq \cdots \geq t_{[n]}$ is a rearrangement of $t_{1} t_{2}, \cdots, t_{n}$ in decreasing order.

Proof. The first statement follows from [2]. For the second, by a result of Fan and Pall [33], $b_{1} \geq b_{2} \cdots \geq b_{m}$ are the eigenvalues of $U^{*} A U$ for some $n \times m$ matrix $U$ satisfying $U^{*} U=I_{m}$ if and only if

from which the result follows.

$$
a_{i} \geq b_{i} \geq a_{n-m+i} \quad \forall 1 \leq i \leq m
$$

Remark 4.2. ( $i)$ If we require the components $\left(t_{1}, \ldots, t_{p}\right)$ in $\Lambda_{(k: p)}(A)$ to be in decreasing order, then the "ordered" $\Lambda_{(k: p)}(A)$ is convex.

(ii) $\Lambda_{k p}(A)=\left[a_{n+1-k p}, a_{k p}\right]$ is obtained by taking the convex hull of the eigenvalues of $A$ after deleting the $(n-k p+1)$ largest and smallest eigenvalues. The following proposition is a generalization of this result.

Proposition 4.3. Suppose $A_{i}=\operatorname{diag}\left(a_{1}^{i}, a_{2}^{i}, \ldots, a_{n}^{i}\right)$ for $i=1, \ldots, m$ with $a_{j}^{i} \in \mathbb{R}$. Let $\mathbf{a}_{j}=$ $\left(a_{j}^{1}, a_{j}^{2}, \ldots, a_{j}^{m}\right)$ for $j=1, \ldots, n$. For $S \subseteq\{1, \ldots, n\}$, let $X_{S}=\operatorname{conv}\left\{\mathbf{a}_{j}: j \in S\right\}$. Then for every $1 \leq k \leq n$,

$$
\Lambda_{k}(\mathbf{A}) \subseteq \cap\left\{X_{S}: S \subset\{1,2, \ldots, n\},|S|=n-k+1\right\} .
$$

Proof. It suffices to prove that $\Lambda_{k}(\mathbf{A}) \subset X_{S}$ for $S=\{1,2, \ldots, n-k+1\}$. Suppose we have $\mathbf{x}=\left(x_{1}, x_{2} \ldots, x_{m}\right) \in \Lambda_{k}(\mathbf{A})$. Then there exists a rank $k$ projection $P$ such that $P A_{i} P=x_{i} P$ for $i=1, \ldots, m$. Consider the subspace $W=\operatorname{span}\left\{e_{1}, \ldots, e_{n-k+1}\right\}$. Then there exists a unit vector $\mathbf{w}=\left(w_{1}, \ldots, w_{n}\right)^{t} \in \mathbb{R}^{n}$ such that $P w=\mathbf{w}$. Therefore, for $1 \leq i \leq m$,

$$
x_{i}=x_{i} \mathbf{w}^{*} \mathbf{w}=x_{i} \mathbf{w}^{*} P \mathbf{w}=\mathbf{w}^{*} P A_{i} P \mathbf{w}=w^{*} A_{i} \mathbf{w}=\sum_{j=1}^{n-k+1}\left|w_{j}\right|^{2} a_{j}^{i \prime} .
$$

Hence, $\mathbf{x}=\sum_{j=1}^{n-k+1}\left|w_{j}\right|^{2} \mathbf{a}_{j} \in X_{S}$.

By the result in [6], equality holds in (8) for $m=1,2$. For $m>2, \Lambda_{k}(\mathbf{A})$ may not be convex and equality may not hold.

Proposition 4.4. Let $A_{i}, 1 \leq i \leq m$ be as given in Proposition 4.3. Then we have:

(1) If $n \geq(m+1) k-m$, then $\Lambda_{k}(\mathbf{A}) \neq \emptyset$. The bound $(m+1) k-m$ is best possible; i.e., if $n<(m+1) k-m$, there exist real diagonal matrices $A_{1}, \ldots, A_{m}$ such that $\Lambda_{k}(\mathbf{A})=\emptyset$.

(2) If $n \geq p((m+1) k-m)$, then $\Lambda_{(k: p)}(\mathbf{A}) \neq \emptyset$.

Proof. The statement (1) follows from Tverberg's Theorem [40] and Proposition 4.3, (Also, see Example 4.6.)

For $(2)$, note that if $n \geq p((m+1) k-m)$, we can decompose each $A_{i}=\oplus_{j=1}^{p} A_{i}^{j}$ with $A_{i}^{j} \in M_{n_{j}}$, and $n_{j} \geq(m+1) k-m$. Then, by the result in 1$), \Lambda_{k}\left(A_{1}^{j}, \ldots, A_{m}^{j}\right) \neq \emptyset$ and the result follows.

Remark 4.5. By the above proposition, for $p((m+1) k-m) \leq n<(m+1) k p-m$, we can construct $A_{1}, \ldots, A_{m}$ such that $\Lambda_{k p}(\mathbf{A})=\emptyset$ and $\Lambda_{(k: p)}(\mathbf{A}) \neq \emptyset$. 
Example 4.6. Suppose $p((m+1) k-m) \leq n<(m+1) k p-m$. We are going to show that there exist $A_{1}, \ldots, A_{m}$ such that $\Lambda_{k p}(\mathbf{A})=\emptyset$ and $\Lambda_{(k: p)}(\mathbf{A}) \neq \emptyset$.

Let $r=\left[\frac{n}{k p-1}\right]$, the greatest integer $\leq \frac{n}{k p-1}$. Then $1 \leq r \leq(m+1)$ and $r=m+1$ if and only if $n=(m+1)(k p-1)$. Define for $1 \leq i \leq \min \{r, m\}, A_{i}=\operatorname{diag}\left(a_{1}^{i}, a_{2}^{i}, \ldots, a_{n}^{i}\right)$, where $a_{i}^{j}=1$ for $(i-1)(k p-1)+1 \leq j \leq i(k p-1)$ and $a_{i}^{j}=0$ otherwise. Then, by Proposition 4.3 , $\Lambda_{k p}(\mathbf{A})=\emptyset$. Since $n \geq p((m+1) k-m)$, by Proposition 4.4, $\Lambda_{(k: p)}(\mathbf{A}) \neq \emptyset$.

\section{OUTLOOK}

As mentioned in Remark 2.5 (vi), one can further extend the definition of $\Lambda_{(k: p)}(\mathbf{A})$ and consider $\left(B_{1}, \ldots, B_{m}\right) \in M_{p}^{m}$ such that $V^{*} A_{j} V=B_{j} \otimes I_{k}$ for some $n \times p k$ matrix $V$ satisfying $V^{*} V=I_{p k}$ without requiring $B_{1}, \ldots, B_{m}$ to be diagonal matrices as in Definition 2.1. We can then use the recent results and techniques in [10] to show that this set is non-empty and star-shaped if $n$ is sufficiently large. This generalization also has a potential implication to the study of quantum error correcting codes. In particular, one may use random qubits to do the encoding and protect the data bits in the quantum error correction process. We plan further research in this direction.

It has been proved that transmitting classical and quantum information simultaneously provides advantages from an information-theoretic perspective [25]. Practical hybrid classical-quantum error correcting codes built on the mathematical techniques introduced here that achieve these advantages could benefit various quantum communication tasks. Communication protocols based on such hybrid codes are expected to enhance the communication security or increase channel capacities. We leave these lines of investigation for future studies.

Recently, the theory of QEC, and especially the framework of OAQEC, has been found to be closely related to the AdS/CFT correspondence and holographic principle in various ways [41, 42, 43, 44, 45, 46]. For instance, Almheiri, Dong and Harlow interpret the complex dictionary in AdS/CFT as the encoding operations of certain operator algebra quantum error correcting codes and bulk local operators are logic operators for these error correcting codes [41. At the same time, holographic codes also inspire new code design methods from a geometric perspective [46]. The matricial range approach to hybrid codes introduced here could conceivably generate new connections between QEC and the theory of quantum gravity.

Acknowledgements. D.W.K. was supported by NSERC. C.K.L. is an affiliate member of the Institute for Quantum Computing, University of Waterloo. His research was supported by USA NSF grant DMS 1331021, and Simons Foundation Grant 351047. M.N. was supported by Mitacs and the African Institute for Mathematical Sciences. B.Z. was supported by NSERC.

\section{REFERENCES}

[1] Man-Duen Choi, David W Kribs, and Karol Zyczkowski. Quantum error correcting codes from the compression formalism. Reports on Mathematical Physics, 58(1):77-91, 2006.

[2] Man-Duen Choi, David W Kribs, and Karol Życzkowski. Higher-rank numerical ranges and compression problems. Linear Algebra and its Applications, 418(2-3):828-839, 2006.

[3] Hugo J Woerdeman. The higher rank numerical range is convex. Linear and Multilinear Algebra, 56(1-2):65-67, 2008.

[4] Chi-Kwong Li, Yiu-Tung Poon, and Nung-Sing Sze. Higher rank numerical ranges and low rank perturbations of quantum channels. Journal of Mathematical Analysis and Applications, 348(2):843-855, 2008.

[5] Man-Duen Choi, Michael Giesinger, John A Holbrook, and David W Kribs. Geometry of higher-rank numerical ranges. Linear and Multilinear Algebra, 56(1-2):53-64, 2008.

[6] Chi-Kwong Li and Nung-Sing Sze. Canonical forms, higher rank numerical ranges, totally isotropic subspaces, and matrix equations. Proceedings of the American Mathematical Society, 136(9):3013-3023, 2008.

[7] Chi-Kwong Li, Yiu-Tung Poon, and Nung-Sing Sze. Condition for the higher rank numerical range to be nonempty. Linear and Multilinear Algebra, 57(4):365-368, 2009. 
[8] David W Kribs, Aron Pasieka, Martin Laforest, Colin Ryan, and Marcus Silva. Research problems on numerical ranges in quantum computing. Linear and Multilinear Algebra, 57(5):491-502, 2009.

[9] Chi-Kwong $\mathrm{Li}$ and Yiu-Tung Poon. Generalized numerical ranges and quantum error correction. Journal of Operator Theory, pages 335-351, 2011.

[10] Pan-Shun Lau, Chi-Kwong Li, Yiu-Tung Poon, and Nung-Sing Sze. Convexity and star-shapedness of matricial range. Journal of Functional Analysis, 275(9):2497-2515, 2018.

[11] Markus Grassl, Sirui Lu, and Bei Zeng. Codes for simultaneous transmission of quantum and classical information. In Information Theory (ISIT), 2017 IEEE International Symposium on, pages 1718-1722. IEEE, 2017.

[12] Andrew Nemec and Andreas Klappenecker. Hybrid codes. In Information Theory Proceedings (ISIT), 2018 IEEE International Symposium on, pages 796-800. IEEE, 2018.

[13] Cédric Bény, Achim Kempf, and David W Kribs. Generalization of quantum error correction via the Heisenberg picture. Physical Review Letters, 98(10):100502, 2007.

[14] Cédric Bény, Achim Kempf, and David W Kribs. Quantum error correction of observables. Physical Review A, 76(4):042303, 2007.

[15] Emanuel Knill, Raymond Laflamme, and Lorenza Viola. Theory of quantum error correction for general noise. Physical Review Letters, 84(11):2525, 2000.

[16] Peter W Shor. Scheme for reducing decoherence in quantum computing memory. Physical Review A, 52:R2493, 1995.

[17] Andrew M Steane. Error correcting codes in quantum theory. Physical Review Letters, 77(5):793, 1996.

[18] Daniel Gottesman. Class of quantum error-correcting codes saturating the quantum Hamming bound. Physical Review A, 54:1862, 1996.

[19] Charles H Bennett, David P DiVincenzo, John A Smolin, and William K Wootters. Mixed state entanglement and quantum error correction. Physical Review A, 54:3824, 1996.

[20] Emanuel Knill and Raymond Laflamme. Theory of quantum error-correcting codes. Physical Review A, 55:900, 1997.

[21] Greg Kuperberg. The capacity of hybrid quantum memory. IEEE Transactions on Information Theory, 49(6):1465-1473, 2003.

[22] David Kribs, Raymond Laflamme, and David Poulin. Unified and generalized approach to quantum error correction. Physical Review Letters, 94(18):180501, 2005.

[23] David W Kribs, Raymond Laflamme, David Poulin, and Maia Lesosky. Operator quantum error correction. Quantum Information E Computation, 6:383-399, 2006.

[24] Cédric Bény, Achim Kempf, and David W Kribs. Quantum error correction on infinite-dimensional Hilbert space. Journal of Mathematical Physics, 50:062108, 2009.

[25] Igor Devetak and Peter W Shor. The capacity of a quantum channel for simultaneous transmission of classical and quantum information. Communications in Mathematical Physics, 256(2):287-303, 2005.

[26] Min-Hsiu Hsieh and Mark M Wilde. Entanglement-assisted communication of classical and quantum information. IEEE Transactions on Information Theory, 56(9):4682-4704, 2010.

[27] Jon Yard. Simultaneous classical-quantum capacities of quantum multiple access channels. arXiv preprint quantph/0506050, 2005.

[28] Isaac Kremsky, Min-Hsiu Hsieh, and Todd A Brun. Classical enhancement of quantum-error-correcting codes. Physical Review A, 78(1):012341, 2008.

[29] Samuel L Braunstein, David W Kribs, and Manas K Patra. Zero-error subspaces of quantum channels. In Information Theory Proceedings (ISIT), 2011 IEEE International Symposium on, pages 104-108. IEEE, 2011.

[30] Manas K Patra and Samuel L Braunstein. An algebraic framework for information theory: classical information. IMA Journal of Mathematical Control and Information, 30(2):205-238, 2013.

[31] Ze-Liang Xiang, Sahel Ashhab, JQ You, and Franco Nori. Hybrid quantum circuits: Superconducting circuits interacting with other quantum systems. Reviews of Modern Physics, 85(2):623, 2013.

[32] John Preskill. Quantum computing in the NISQ era and beyond. arXiv preprint arXiv:1801.00862, 2018.

[33] Ky Fan and Gordan Pall. Imbedding conditions for Hermitian and normal matrices. Canadian Journal of Mathematics, 9:298-304, 1957.

[34] Hwa-Long Gau, Chi-Kwong Li, Yiu-Tung Poon, and Nung-Sing Sze. Higher rank numerical ranges of normal matrices. SIAM Journal of Matrix Analysis and its Applications, 32:23-43, 2011.

[35] Man-Duen Choi. Completely positive linear maps on complex matrices. Linear Algebra and its Applications, 10(3):285-290, 1975.

[36] Michael A Nielsen and Isaac L Chuang. Quantum Computation and Quantum Information, 2000.

[37] Kenneth R Davidson. C*-algebras by example, volume 6. American Mathematical Soc., 1996.

[38] Chi-Kwong Li, Mikio Nakahara, Yiu-Tung Poon, Nung-Sing Sze, and Hiroyuki Tomita. Efficient quantum error correction for fully correlated noise. Physics Letters A, 375(37):3255-3258, 2011. 
[39] Chi-Kwong Li, Seth Lyles, and Yiu-Tung Poon. Error correction schemes for fully correlated quantum channels protecting both quantum and classical information. https://arxiv.org/pdf/1905.10228.

[40] Helge Tverberg. A generalization of Radon's theorem. Journal of London Mathematics Society, 41:123-128, 1966.

[41] Ahmed Almheiri, Xi Dong, and Daniel Harlow. Bulk locality and quantum error correction in AdS/CFT. Journal of High Energy Physics, 2015(4):163, 2015.

[42] Fernando Pastawski, Beni Yoshida, Daniel Harlow, and John Preskill. Holographic quantum error-correcting codes: Toy models for the bulk/boundary correspondence. Journal of High Energy Physics, 2015(6):149, 2015.

[43] Daniel Harlow. The ryu-takayanagi formula from quantum error correction. Communications in Mathematical Physics, 354(3):865-912, 2017.

[44] Fabio Sanches and Sean J Weinberg. Holographic entanglement entropy conjecture for general spacetimes. Physical Review D, 94(8):084034, 2016.

[45] Leander Fiedler, Pieter Naaijkens, and Tobias J Osborne. Jones index, secret sharing and total quantum dimension. New Journal of Physics, 19(2):023039, 2017.

[46] Fernando Pastawski and John Preskill. Code properties from holographic geometries. Physical Review X, $7(2): 021022,2017$.

${ }^{1}$ Department of Mathematics \& Statistics, University of Guelph, Guelph, On, Canada N1G 2W1

${ }^{2}$ Institute for Quantum Computing, University of Waterloo, Waterloo, ON, Canada N2L 3G1

${ }^{3}$ Department of Mathematics, College of William \& Mary, Williamsburg, VA, USA 23187

${ }^{4}$ Department of Mathematics, Iowa State University, Ames, IA, USA 50011

${ }^{5}$ Department of Physics, The Hong Kong University of Science and Technology, Clear Water Bay, KOWlOON, HONG KONG

${ }^{6}$ Center for Quantum Computing, Peng Cheng Laboratory, Shenzhen, 518055, China 